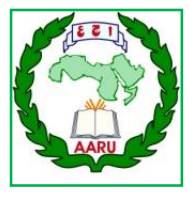

Arab Univ. J. Agric. Sci., Ain Shams Univ., Cairo, Egypt

29(2), 611-626, 2021

Website: http://ajs.journals.ekb.eg

DOI: 10.21608/ajs.2021.54290.1317

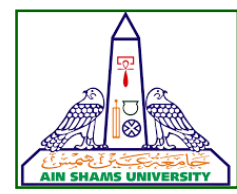

611

\title{
Evaluation of Some Transpiration Regulators in Sunflower for Saving Irrigation Water in Relation to Productivity
}

\section{Naglaa MT Soliman ${ }^{1 *}$, Ayman F Abou-Hadid ${ }^{2}$, Mohamed TB Fayed ${ }^{3}$, Mohamed AH Fergany ${ }^{3}$}

1- Oil Crops Research Dept, Agronomy Research Institute, Agricultural Research Center, Giza, Egypt

2- Horticulture Dept, Fac of Agric, Ain Shams Univ, P.O. Box 68, Hadayek Shoubra 11241, Cairo, Egypt

3- Agronomy Dept, Fac of Agric, Ain Shams Univ, P.O. Box 68, Hadayek Shoubra 11241, Cairo, Egypt

*Corresponding author: naglaamahmoud_2004@yahoo.com

Received 20 February, 2021

Accepted 19 June, 2021

\begin{abstract}
An experimental field trial was conducted in seasons of 2015 and 2016 at Giza Experimental Station of Agricultural Research Centre (ARC), Egypt to evaluate the foliar spray effect with transpiration regulators (Potassium, Kaolin and Ascorbic acid) and three levels of irrigations $\left(100 \%, 80 \%\right.$ and $60 \%$ of $\left.\mathrm{Et}_{0}\right)$ on yield, its attributes, and water productivity of two sunflower cultivars (Giza 102 and Solala 120). The factorial treatments were arranged in split- split plot design with three replications. The irrigation levels $(100 \%, 80 \%$ and $60 \%$ of $\mathrm{Et}_{0}$ ) were devoted for main plots, while subplots contained the three spraying treatments of transpiration regulators. (Potassium at 1\%, Kaolin at 3\% and Ascorbic acid at 100 ppm), in addition to control treatment (water spray). Sub-sub plots were occupied by the two sunflower cultivars (Giza 102 and Solala 120). Obtained results proved that, increasing irrigation levels up to (80-100\%) caused significant increase in all growth characteristics, photosynthetic pigments (total chlorophyll), relative water content (RWC \%) and seed oil \%. Also, yield parameters being head diameter, 1000-
\end{abstract}

seed weight and seed yield) show significant reduction under water deficit. Foliar spray with (Potassium at 1\%, Kaolin at 3\% and Ascorbic acid at $100 \mathrm{ppm}$ ), positively affected all the growth and physiological criteria of the tested plants compared with control. Generally, under low irrigation levels, application of transpiration regulators effectively reduced the detrimental impact of drought stress on growth, yield and its components of the two used cultivars in the two seasons. While, foliar spraying of potassium under $100 \%$ of $\mathrm{Et}_{0}$ gave the best treatment in this respect. Concerning cultivars, WUE (water use efficiency)for Solala 120 was significantly higher than for Giza 102 in the two seasons. All interactions among factors under study due to WUE were not significant in 2015 and 2016 seasons.

Keywords: Sunflower, Drought, Water use efficiency, Potassium, Ascorbic acid, Kaolin

\section{Introduction}

The sunflower (Helianthus annuus L.) is main crop as a source for vegetable oil that is unsaturated. It produced about $35,000,000$ tons 
per year from cultivated area ranged about $25,000,000 \mathrm{ha}$. Sunflower is the main grower crop in Europe and produced about $65 \%$ of the world's total production in 2010. Albert and Schneiter (1997) mentioned that sunflower is one of few crop species originating in North America and spread to western and drier climates areas.

Liux and Baird (2003) explained that today, Argentina, Eastern European, and the former Soviet Union are the main producers of sunflower crop. Due to its intermediate drought and salinity tolerance, its cultivation increased in the arid regions of North Africa (Miller, 1995, Connor and Hall 1997). Seeds of sunflower characterized by a high percent of unsaturated fatty acids containing more than $90 \%$ linoleic (18:2) and oleic (18:1) acids with possible health benefits as indicated by (LopezPereira et al 2000, Leon et al 2003). The corner stone for determining the composition of fatty acid, oil \% and unsaturated fatty acids is genotype. Oil composition is also influenced by environmental conditions at all growth stages of plant (Knowles 1988).

Karam et al (2007) elucidated that sunflower crop is characterized by Photo insensitive property, so it is grown as rain fed crop throughout the year in many parts of the world but responds positively to irrigation in terms of growth and yield, where there is limited precipitation and soil water supply. As a response to applied irrigation, sunflower is tolerant to moderate water stress and able to bear high yield.

Hoque et al (2016) concluded that most plants continuously exposed to one or many abiotic stresses (such as, high temperatures, shortage water, and salinity) can restrict plant growth and yield.

The adverse effects of many stresses on plants in nature are decreasing food productivity, thus, reducing these damages is a basic goal to ensure food security under climate change (Anjum et al 2011).

Transpiration regulators are chemical compounds whose function is helping plants to resist the bad effect of drought by gradually hardening them to stress. There are various forms of antitranspirants, among them, filmforming, which prevents almost all transpiration and stomatic which affects only the stomata (Nasraui 1993).

Prakash and Ramachandran (2000) indicated that the transpiration regulators are materials or chemical compounds that reducing the water losses from plant leaves by decreasing the size as well as number of stomata. Almost $99 \%$ of the absorbed water by plant is lost during transpiration. It is probably to classify transpiration regulators into three types, namely film-forming types (coating the surface of the leaves with films that tries to prevent water vapor), stomatal closure (which affect the metabolic processes in leaf tissues), and reflecting materials (reflecting a portion of the incident radiation that falls on the upper surface of the leaves). Umar and Moinuddin (2002) concluded that potassium is one of the most important macronutrients, motivates growth of plants, decrease transpiration and increases the maintenance of water plants. Ascorbate has been play multiple roles in plant growth, such as in cell division, cell wall expansion, and other developmental processes. Antitranspirant are substances involved in increasing drought resistance by tending to cause xeromorphy and/or stabilizing cell structure (Ouda et al 2007). Kaolin application has been recently recommended for sustainable viticulture for wine growers to meet various challenges of changing climatic conditions, especially in Mediterranean-like climates (Cond 2016).

This work aimed to investigate the effect of water deficit on growth, seed and oil yields of sunflower plants. Also, to evaluate activity of some transpiration regulators in saving irrigation water in relation to productivity.

\section{Materials and Methods}

An experimental field trial was conducted in the two seasons of 2015 and 2016 at Giza Experimental Research Station ARC, Egypt to evaluate the efficiency of some transpiration regulators in sunflower plant for saving irrigation water in relation to productivity. Each trial 
was designed in split- split plot arrangements and replicates three times. The three irrigation levels i.e. $100 \%, 80 \%$ and $60 \%$ of $\mathrm{Et}_{0}$ (The rate of evapotranspiration from an excessive surface of green cover of uniform height ( 8 to 15 $\mathrm{cm}$ ), actively growing, completely shading the ground and did not suffer water shortage), whereas subplots randomly contained three spraying treatments of antitranspirant agents (Potassium 1\%, Kaolin 3\% and Ascorbic acid at $100 \mathrm{ppm}$, in addition to control (water spray). Sub-sub plots were occupied by the two cultivars (Giza 102 and Solala 120). Soil samples were randomly taken from experimental field at depth of 0 to $30 \mathrm{~cm}$ from soil surface and were analyzed for physical and chemical characteristics according to Israelsen and Hansen (1962). The mechanical and chemical analysis of soil samples are presented in (Table 1).

\subsection{Studied characters}

\subsubsection{Leaves area/plant (LA), in $\mathrm{cm}^{2}$}

According to method of Johnson (1967), ten dried leaves disc were weighed and measure their area, then the leaf area per plant was estimated from the following formula:

LA/ plant $\left(\mathrm{cm}^{2}\right)=$ Leaf dry weight $\times$ disc area/Disc dry weight.

\subsubsection{Chlorophyll content in leaves}

The total chlorophyll pigments were determined by reading the absorbance on spectrophotometer at 664 and $647 \mathrm{~nm}$ and concentration of photosynthetic pigments were calculated according to the equation mentioned by Moran (1982).

\subsubsection{Leaf relative water content (LRWC\%)}

Relative water content in sunflower leaves was determined according to the method of Weatherly (1950) and its modification by
Barrs and Weatherly (1962), and the consideration given by El-Sharkawy and Salama (1973). Leaf discs, were punched from the center of the leaf. Fresh weight was taken (FW) and floated for 4 hours in distilled water and weighed again to obtain turgid weight (TW). For dry weight (DW) determination, the discs were weighed and relative water content was calculated according to the following equation:

$$
\mathrm{RWC}(\%)=(\mathrm{FW}-\mathrm{DW}) /(\mathrm{TW}-\mathrm{DW}) \times 100
$$

\subsubsection{Head diameter $(\mathrm{cm})$}

\subsubsection{Plant height (cm)}

\subsubsection{0- seed weight $(g)$}

Three random samples of 100 seeds were weighed from each sub- sub plot. The means of the three samples were multiplied by 10 .

\subsubsection{Seed yield/fed (kg)}

As the total seed weight from $3 \mathrm{~m}^{2}$ (25 plants) and converted to seed yield/fed.

\subsubsection{Oil content \%}

Oil content $\%$ of sunflower seeds was determined according to AOAC (1980) using Soxhlet apparatus and petroleum ether as a solvent.

\subsubsection{Oil yield/ fed (kg)}

Determined by multiplying seed yield (kg/fed.) by seed oil percentage.

\subsubsection{Water Use Efficiency (WUE)}

Water use efficiency in $\mathrm{kg} / \mathrm{m}^{3} / \mathrm{fed}$. was calculated for each treatment according to the equation given by Jensen (1983) as follow:

WUE $=($ Seed yield $(\mathrm{kg} / \mathrm{fed})) /.($ Seasonal water consumption $\left(\mathrm{m}^{3} / \mathrm{fed}.\right)$ ). 
Table 1. The mechanical and chemical analysis of the experimental soil

\begin{tabular}{|c|c|c|c|c|c|c|c|c|c|c|c|c|c|}
\hline \multicolumn{3}{|c|}{ Coares sand\% } & \multicolumn{3}{|c|}{ Fine sand\% } & \multicolumn{3}{|c|}{ Silt \% } & \multicolumn{3}{|c|}{ Clay \% } & \multicolumn{2}{|c|}{ Soil texture } \\
\hline \multicolumn{3}{|c|}{1.5} & \multicolumn{3}{|c|}{31.9} & \multicolumn{3}{|c|}{29.8} & \multicolumn{3}{|c|}{34.7} & \multicolumn{2}{|c|}{ Sandy loam } \\
\hline \multicolumn{10}{|c|}{ Cations (ppm) } & \multicolumn{2}{|c|}{ Anions (ppm) } & \multirow{2}{*}{ PH } & \multirow{2}{*}{$\mathrm{EC}(\mathrm{ds} / \mathrm{m})$} \\
\hline $\mathrm{Ca}++$ & $\mathrm{Mg++}$ & $\mathrm{N}$ & $\mathrm{P}$ & $\mathrm{K}+$ & $\mathrm{Na}+$ & $\mathrm{Cu}+$ & $\mathrm{Zn+}$ & $\mathrm{Mn+}$ & $\mathrm{Fe}++$ & $\mathrm{Cl}$ & $\mathrm{Caco} 3$ & & \\
\hline 4.4 & 1.6 & 33.3 & 8.4 & 129 & 3.7 & 0.96 & 0.91 & 4.1 & 3.1 & 2.2 & 0.8 & 7.4 & 1.8 \\
\hline
\end{tabular}

\subsection{Statistical analysis}

All statistical analyses were automated according to Steel and Torrie (1980). Least significant difference at $5 \%$ level of probability was used to compare between mean of treatments.

\section{Results and Discussion}

\subsection{Leaf area}

Leaf area/plant under the various treatments is recorded in (Table 2). Water irrigation levels significantly affected leaf area/plant in the two seasons. Irrigation of $60 \%$ gave the lowest value of leaf area/plant. Interpretation of such finding was reported by Yegappan et al (1982) who found that under limited irrigation conditions full expansion of leaves, cell number and leaf area were decreased. They added that these results help to explain the effect of water stress on reduce leaf area especially the reduce of the lower leaves that are the most sensitive to water stress. Furthermore, Attia (1985) found that water deficit caused a depression in the area of leaves. He added that high soil moisture not only favors cell division and expansion but also enhanced the production of photosynthetic surface of sunflower plants.

Significant difference between the tested cultivars was recorded for leaf area/plant. Solala 120 gave the higher values in both seasons. Similar results were obtained by (Demian 1999).

Concerning transpiration regulators, leaf area/plant significantly affected by the applied transpiration regulators in 2015 and 2016 seasons. Leaf area of treated plants surpassed the control (nonatitrancpirant agents) without significant differences among them. These results agree with those obtained by agreement with those of (Ismail and Hasabo 2000, Sherif 2002, El-Temsah 2008).

All interaction effects among the studied factors were not significant for leaves area/plant in both seasons.

\section{3-2 Leaf relative water content (LRWC \%)}

Relative water content values of sunflower leaves were significantly governed by soil moisture \%. The highest LRWC\% values were recorded with the $100 \%$ of $\mathrm{Et}_{0}$ followed by the $80 \%$ of $\mathrm{Et}_{0}$. However, the lowest LRWC\% value was obtained from irrigation at dry treatment under $60 \%$ of level water treated with Ascorbic acid. Such finding was true in both seasons. The results demonstrated that, plant water relations play a pivotal job in maintaining the physiological advantages of sunflower plants. Monroy et al (2015) Reported that, the treatment of adequate irrigation had higher RWC\% than water stressed treatment. Also Unyayar et al (2004) stated that RWC\% of the sunflower leaves decreased under drought stress. Since, in sunflowers plants water stress caused significant decrease in relative water content \%. (El-Mantawy et al 2017).

It is obvious from the results of (Table 2) that spraying sunflower plants with Potassium Under $100 \%$ of irrigation water in both seasons (2015-2016) significantly increased LRWC\% as compared with untreated plants. transpiration regulators which form a film on the surface of sunflower plants increase the (LRWC $\%$ ) in leaves especially at water stress (AbdelFatah, 2013). 

Irrigation Water in Relation to Productivity

Table 2. Effect of irrigation and transpiration regulators on leaf area (LA) $\left(\mathrm{cm}^{2}\right)$ and relative water content (RWC \%) of two sunflower cultivars in 2015 and 2016 seasons

\begin{tabular}{|c|c|c|c|c|c|c|c|c|c|c|c|c|c|}
\hline \multirow{4}{*}{ Irrigation } & \multirow{4}{*}{ Treatments } & \multicolumn{6}{|c|}{ Leaf area $(\mathrm{LA})(\mathrm{cm} 2)$} & \multicolumn{6}{|c|}{ Relative water content (RWC \%) } \\
\hline & & \multicolumn{3}{|c|}{$1^{\text {st }}$ season (2015) } & \multicolumn{3}{|c|}{$2^{\text {nd }} \operatorname{season}(2016)$} & \multicolumn{3}{|c|}{$1^{\text {st }}$ season (2015) } & \multicolumn{3}{|c|}{$2^{\text {nd }}$ season (2016) } \\
\hline & & \multicolumn{2}{|c|}{ Cultivar } & \multirow{2}{*}{\begin{tabular}{|l|} 
Mean \\
\end{tabular}} & \multicolumn{2}{|c|}{ Cultivar } & \multirow{2}{*}{\begin{tabular}{|l|} 
Mean \\
\end{tabular}} & \multicolumn{2}{|c|}{ Cultivar } & \multirow[t]{2}{*}{ Mean } & \multicolumn{2}{|c|}{ Cultivar } & \multirow[t]{2}{*}{ Mean } \\
\hline & & $\begin{array}{c}\text { Giza } \\
(102)\end{array}$ & \begin{tabular}{|c|} 
Solala \\
$(\mathbf{1 2 0})$
\end{tabular} & & \begin{tabular}{c|} 
Giza \\
$(102)$
\end{tabular} & \begin{tabular}{|c|} 
Solala \\
$(120)$
\end{tabular} & & \begin{tabular}{c|} 
Giza \\
$(102)$ \\
\end{tabular} & \begin{tabular}{|c|} 
Solala \\
$(120)$
\end{tabular} & & \begin{tabular}{|l|} 
Giza \\
$(102)$ \\
\end{tabular} & $\begin{array}{c}\text { Solala } \\
(120)\end{array}$ & \\
\hline \multirow{4}{*}{$60 \%$} & Control & 2039 & 2368 & 2204 & 1960 & 2748 & 2354 & 45.5 & 57.0 & 51.3 & 48.8 & 60.3 & 54.5 \\
\hline & Potassium & 2260 & 2587 & 2424 & 2166 & 2954 & 2560 & 56.6 & 68.1 & 62.3 & 59.8 & 71.3 & 65.6 \\
\hline & Kaolin & 2220 & 2547 & 2384 & 2129 & 2917 & 2523 & \begin{tabular}{|l|}
52.4 \\
\end{tabular} & 63.9 & 58.2 & 54.7 & 66.2 & 60.4 \\
\hline & Ascorbic & 2197 & 2524 & 2361 & 2111 & 2899 & 2505 & \begin{tabular}{|l|}
50.4 \\
\end{tabular} & 61.1 & 55.8 & 52.3 & 63.8 & 58.1 \\
\hline Mean & & 2179 & 2507 & 2343 & 2092 & 2879 & 2486 & \begin{tabular}{|l|}
51.2 \\
\end{tabular} & 62.5 & 56.9 & \begin{tabular}{|l|}
53.9 \\
\end{tabular} & 65.41 & 59.7 \\
\hline \multirow{4}{*}{$80 \%$} & Control & 2582 & \begin{tabular}{|l|}
2909 \\
\end{tabular} & 2746 & \begin{tabular}{|l|}
2486 \\
\end{tabular} & \begin{tabular}{|l|}
3068 \\
\end{tabular} & \begin{tabular}{|l|}
2777 \\
\end{tabular} & \begin{tabular}{|l|}
50.4 \\
\end{tabular} & \begin{tabular}{|l|}
61.4 \\
\end{tabular} & 55.9 & \begin{tabular}{|l|}
51.9 \\
\end{tabular} & 62.9 & \begin{tabular}{|l|}
57.4 \\
\end{tabular} \\
\hline & Potassium & 2801 & 3128 & 2965 & 2719 & 3274 & 2997 & 61.4 & 72.4 & 66.9 & 62.7 & 73.9 & 68.3 \\
\hline & Kaolin & 2761 & 3088 & 2925 & 2655 & 3237 & 2946 & 57.3 & 68.3 & 62.8 & 58.8 & 69.8 & 64.3 \\
\hline & Ascorbic & 2738 & 3065 & 2902 & 2637 & 3219 & 2928 & 54.3 & 65.3 & 59.8 & 55.8 & 66.8 & 61.3 \\
\hline Mean & & 2721 & 3048 & 2884 & 2593 & 3199 & 2896 & 55.8 & \begin{tabular}{|l|}
66.8 \\
\end{tabular} & 61.3 & \begin{tabular}{|l|}
57.3 \\
\end{tabular} & 68.3 & \begin{tabular}{|l|}
62.8 \\
\end{tabular} \\
\hline \multirow{4}{*}{$100 \%$} & Control & 2805 & 3053 & 2929 & 2624 & 3206 & 2915 & 58.8 & 65.7 & 62.3 & 56.6 & 67.3 & 62.0 \\
\hline & Potassium & 2965 & \begin{tabular}{|l|}
3292 \\
\end{tabular} & 3129 & 2828 & \begin{tabular}{|l|}
3412 \\
\end{tabular} & 3120 & \begin{tabular}{|l|}
66.1 \\
\end{tabular} & \begin{tabular}{|l|}
76.8 \\
\end{tabular} & 71.4 & \begin{tabular}{|l|}
67.7 \\
\end{tabular} & 78.7 & 73.2 \\
\hline & Kaolin & 2916 & 3244 & 3080 & 2791 & 3375 & 3083 & \begin{tabular}{|l|}
61.9 \\
\end{tabular} & 72.6 & 67.3 & 63.5 & 74.2 & 68.9 \\
\hline & Ascorbic & 1290 & 3217 & 2254 & 2775 & 3357 & 3066 & \begin{tabular}{|l|}
59.1 \\
\end{tabular} & 71.0 & 65.0 & \begin{tabular}{|l|}
60.5 \\
\end{tabular} & 71.2 & 65.9 \\
\hline Mean & & 2494 & 3202 & 2848 & 2755 & 3337 & 3046 & 61.5 & 71.5 & 66.5 & 62.1 & 72.9 & 67.5 \\
\hline \multicolumn{14}{|l|}{ LSD } \\
\hline $\mathrm{V}$ & & & & 0.24 & & & 0.30 & & & 1.72 & & & 0.07 \\
\hline $\mathrm{Ir}$ & & & & 0.19 & & & 0.25 & & & 1.54 & & & 0.06 \\
\hline $\mathrm{V} * \mathrm{ir}$ & & & & \begin{tabular}{|l|}
0.27 \\
\end{tabular} & & & \begin{tabular}{|l|}
0.30 \\
\end{tabular} & & & 0.06 & & & 0.09 \\
\hline $\mathrm{Tr}$ & & & & 0.20 & & & \begin{tabular}{|l|}
0.25 \\
\end{tabular} & & & 1.60 & & & \begin{tabular}{|l|}
0.06 \\
\end{tabular} \\
\hline Tr*ir & & & & 0.28 & & & 0.30 & & & n.s & & & n.s \\
\hline $\mathrm{T}^{*} \mathrm{~V}$ & & & & 0.34 & & & 0.36 & & & n.s & & & n.s \\
\hline $\mathrm{Tr}^{*} \mathrm{ir}^{*} \mathrm{~V}$ & & & & 0.48 & & & 0.50 & & & n.s & & & n.s \\
\hline
\end{tabular}

$\mathrm{V}$ : Cultivar IR: irrigation levels

\subsection{Plant height $(\mathrm{cm})$}

Data in (Table 3) cleared that water stress $\left(60 \% \mathrm{Et}_{0}\right)$ treatment sharply decreased sunflower plant height in both seasons. Contrarily, plant height was increased as irrigation water levels increased. Depression in plant height under drought stress may be due to the retardation in cell division and cell expansion caused by water stress (Attia 1985). These results are in harmony with those obtained by (Wahba et al 1990, Khan et al 2000, Ahmed et al 2009). As for cultivars, Giza 102 gave taller plants than Solala 120. Varietal differences in plant height were found also by (Salera and Detti 1992, El-Hity et al 1994).

Height of sunflower plant was positively affected by the application of transpiration regulators. This trend was true in both experimental seasons, but it was more pronounced under drought stress conditions. Under60\% irrigation level, the application of Potassium, Ascorbic and Kaolin transpiration regulators treatments increased height of sunflower plant in the 2 nd season than the control by $4.9 \%$, $3.4 \%$ and $2.6 \%$, respectively. Transpiration 
regulators minimized natural plant transpiration rate and saved cell water to maintain the efficiency of physiological processes in stressed plant.

\subsection{Head diameter $(\mathrm{cm})$}

Data presented in (Table 3) Reveal the influence of irrigation levels and transpiration regulators on head diameter of the two sunflower cultivar plants. It is clear that head diameter trait was significantly affected by irrigation levels. Irrigation at $100 \%$ at $\mathrm{Et}_{0}$ produced the highest values of head diameter $(\mathrm{cm})$ in the two seasons. Otherwise, irrigation at $60 \%$ at $\mathrm{Et}_{0}$ caused a marked reduction in head diameter in 2015 and 2016 seasons. The reduction in vegetative biomass caused by drought performed to lower plant surface area which subsequently limited the photosynthetic activities and radiation use efficiency (Stockle and Kiniry 1990, Badr and Mohamed 2004). This finally decreased net assimilation rate of photosynthetic area during the reproductive phase which reduce head diameter. Reduction in head diameter further decrease, the number of rows per head, number achiness per head (Rauf and Sadaqat 2007).

With respect to cultivars, data indicated that Solala 120 gave higher head diameter as compared with Giza 102 in both seasons. These results are in harmony with those obtained by Demian (1999). Who found that head diameter significantly differed by cultivars.

Results indicated that transpiration regulators significantly surpassed control treatment for head diameter in both seasons. Application of potassium gave the largest head diameter. The interactions among the three studied factors had no significant effect on head diameter of sunflower plants in both seasons.

\subsection{0-seed weight (g)}

Data listed in (Table 4) reveal the irrigation levels and transpiration regulators effect on 1000- seed weight of two sunflower cultivars in the two experimental seasons of 2015 and 2016. Seed index (1000-seed) weight values were significantly affected by irrigation level treatments in the two growing seasons. Irrigation at $100 \%$ of Et $_{0}$ gave the heaviest 1000 seed weight followed by irrigation at $80 \%$ of $\mathrm{Et}_{0}$ in 2015 , with no significant difference. However, in 2016 season irrigation at $100 \%$ of $\mathrm{Et}_{0}$ gave the highest value. In both seasons, irrigation at $60 \%$ of $\mathrm{Et}_{0}$ produced the lowest value of 1000 seed weight. In this respect, Baldini and Vannozzi (1999), Rady et al (2003) reported that water deficit during the flowering stage causes abortion of ovaries, embryo, sterility of pollen and decreased in leaf area index. This reduced the number of achanes/head, 100-achene weight and fertile achene per head. Similar trend was achieved by (Prunty 1983, AbdelGawad et al 1987).

The difference in 1000-seed weight between cultivars was significant in both seasons. Solala 120 Cultivar had heavier 1000seed weight than Giza 102. El-Ahmer et al (1989), Demian (1999) found difference in 1000-seed weight among cultivars, foliar application of transpiration regulators increased in some extents seeds weight of sunflower plants in both experimental seasons. Improvement in such trail was more pronounced under soil water deficit conditions (Table 4). Potassium transpiration regulators was the potent treatment in this respect and increased 1000seed weight was increased by 3.2, 2.7, 2.0\% under $60,80,100 \%$ of water levels in the first season, respectively.

Similar trend values in the $2^{\text {nd }}$ season were 4.7, 2.1 and $0.7 \%$ for the same respective soil moisture levels. Potassium regulates stomatal aperture width through the regulation of osmatic pressure of guard cells. Such mechanism saves relative water content of plant tissues (Table 2) and favors photosynthetic activity and translocation of biosynthetic assimilates to accumulate in the formed seeds.

Seed index (1000-seed weight) of sunflower was governed statistically by the interaction between the studied factors. This trend is true in two seasons. Heaviest sunflower seeds in the two seasons were obtained by Solala 120 cultivar treated with Potassium under $100 \% \mathrm{Et}_{0}$ irrigation level. 


\section{Evaluation of Some Transpiration Regulators in Sunflower for Saving Irrigation Water in Relation to Productivity}

Table 3. Effect of irrigation and transpiration regulators on plant height $(\mathrm{cm})$ and head diameter $(\mathrm{cm})$ of two sunflower cultivars in 2015 and 2016 seasons

\begin{tabular}{|c|c|c|c|c|c|c|c|c|c|c|c|c|c|}
\hline \multirow{4}{*}{ Irrigation } & \multirow{4}{*}{ Treatments } & \multicolumn{6}{|c|}{ Plant height (cm) } & \multicolumn{6}{|c|}{ Head diameter $(\mathrm{cm})$} \\
\hline & & \multicolumn{3}{|c|}{$1^{\text {st }}$ season $(2015)$} & \multicolumn{3}{|c|}{$2^{\text {nd }} \operatorname{season}(2016)$} & \multicolumn{3}{|c|}{$1^{\text {st }} \operatorname{season}(2015)$} & \multicolumn{3}{|c|}{$2^{\text {nd }} \operatorname{season}(2016)$} \\
\hline & & \multicolumn{2}{|c|}{ Cultivar } & \multirow[t]{2}{*}{ Mean } & \multicolumn{2}{|c|}{ Cultivar } & \multirow[t]{2}{*}{ Mean } & \multicolumn{2}{|c|}{ Cultivar } & \multirow[t]{2}{*}{ Mean } & \multicolumn{2}{|c|}{ Cultivar } & \multirow[t]{2}{*}{ Mean } \\
\hline & & $\begin{array}{l}\text { Giza } \\
(102)\end{array}$ & $\begin{array}{c}\text { Solala } \\
(120)\end{array}$ & & $\begin{array}{l}\text { Giza } \\
(102)\end{array}$ & $\begin{array}{c}\text { Solala } \\
(120)\end{array}$ & & $\begin{array}{l}\text { Giza } \\
(102)\end{array}$ & $\begin{array}{c}\text { Solala } \\
(\mathbf{1 2 0})\end{array}$ & & $\begin{array}{c}\text { Giza } \\
(102)\end{array}$ & $\begin{array}{c}\text { Solala } \\
(\mathbf{1 2 0})\end{array}$ & \\
\hline \multirow{4}{*}{$60 \%$} & Control & 160.0 & 118.0 & 139.0 & 145.0 & 116.0 & 130.5 & 15.72 & 20.96 & 18.34 & 15.90 & 20.9 & 18.40 \\
\hline & Potassium & 171.3 & 123.0 & 147.2 & 151.0 & 123.0 & 137.0 & 17.22 & 22.46 & 19.84 & 17.14 & 22.40 & 19.77 \\
\hline & Kaolin & 120.2 & 122.0 & 121.1 & 149.0 & 121.0 & 135.0 & 16.81 & 21.97 & 19.39 & 16.63 & 21.80 & 19.22 \\
\hline & Ascorbic & 169.1 & 121.0 & 145.1 & 148.0 & 120.0 & 134.0 & 16.30 & 21.56 & 18.93 & 16.27 & 21.40 & 18.84 \\
\hline Mean & & 155.2 & 121.0 & 138.1 & 148.3 & 120.0 & 134.1 & 16.51 & 21.74 & 19.13 & 16.49 & 21.63 & 19.06 \\
\hline \multirow{4}{*}{$80 \%$} & Control & 157.1 & 127.0 & 142.1 & 154.0 & 125.0 & 139.5 & 17.86 & 23.2 & 20.53 & 17.50 & 22.80 & 20.15 \\
\hline & Potassium & 162.5 & 132.0 & 147.3 & 160.0 & 131.0 & 145.5 & 19.35 & 24.50 & 21.93 & 19.10 & 24.20 & 21.65 \\
\hline & Kaolin & 161.4 & 131.0 & 146.2 & 159.0 & 130.0 & 144.5 & 18.85 & 23.99 & 21.42 & 18.46 & 23.60 & 21.03 \\
\hline & Ascorbic & 160.2 & 131.0 & 145.6 & 157.0 & 129.0 & 143.0 & 18.44 & 23.62 & 21.03 & 18.10 & 23.30 & 20.70 \\
\hline Mean & & 160.3 & 130.3 & 145.3 & 157.5 & 128.8 & 143.1 & 18.63 & 23.83 & 21.23 & 18.29 & 23.48 & 20.88 \\
\hline \multirow{4}{*}{$100 \%$} & Control & 163.5 & 131.0 & 147.3 & 158.0 & 130.0 & 144.0 & 18.34 & 23.50 & 20.92 & 17.91 & 23.10 & 20.51 \\
\hline & Potassium & 166.5 & 137.0 & 151.8 & 163.0 & 135.0 & 149.0 & 19.86 & 25.00 & 22.43 & 19.37 & 24.60 & 21.99 \\
\hline & Kaolin & 165.4 & 135.0 & 150.2 & 161.0 & 134.0 & 147.5 & 19.37 & 24.55 & 21.96 & 18.44 & 24.40 & 21.42 \\
\hline & Ascorbic & 164.4 & 134.0 & 149.2 & 161.0 & 133.0 & 147.0 & 18.92 & 24.14 & 21.53 & 18.44 & 23.60 & 21.02 \\
\hline Mean & & 165.0 & 134.3 & 149.6 & 160.8 & 133.0 & 146.9 & 19.12 & 24.30 & 21.71 & 18.54 & 23.93 & 21.23 \\
\hline \multicolumn{14}{|l|}{ LSD } \\
\hline V & & & & 0.17 & & & 0.16 & & & 0.22 & & & 0.03 \\
\hline $\mathrm{Ir}$ & & & & 0.13 & & & 0.13 & & & 0.00 & & & 0.03 \\
\hline$V^{*}$ ir & & & & n.s & & & n.s & & & 0.40 & & & 0.06 \\
\hline $\operatorname{Tr}$ & & & & 0.16 & & & 0.15 & & & 0.27 & & & 0.05 \\
\hline Tr*ir & & & & 0.27 & & & 0.26 & & & n.s & & & n.s \\
\hline $\mathrm{T}^{*} \mathrm{~V}$ & & & & n.s & & & n.s & & & n.s & & & n.s \\
\hline Tr*ir*V & & & & n.s & & & n.s & & & n.s & & & n.s \\
\hline
\end{tabular}

V: Cultivar IR: irrigation levels

Tr: treatments 
Table 4. Effect of irrigation and transpiration regulators on 1000-seed weight ( $\mathrm{g}$ ) and seed yield/fed (kg) of two sunflower cultivars in 2015 and 2016 seasons

\begin{tabular}{|c|c|c|c|c|c|c|c|c|c|c|c|c|c|}
\hline \multirow{4}{*}{ Irrigation } & \multirow{4}{*}{ Treatments } & \multicolumn{6}{|c|}{1000 -seed weight $(\mathrm{g})$} & \multicolumn{6}{|c|}{ Seed yield kg/fed } \\
\hline & & \multicolumn{3}{|c|}{$1^{\text {st }}$ season $(2015)$} & \multicolumn{3}{|c|}{$2^{\text {nd }} \operatorname{season}(2016)$} & \multicolumn{3}{|c|}{$1^{\text {st }}$ season $(2015)$} & \multicolumn{3}{|c|}{$2^{\text {nd }}$ season $(2016)$} \\
\hline & & \multicolumn{2}{|c|}{ Cultivar } & \multirow{2}{*}{\begin{tabular}{|l|} 
Mean \\
\end{tabular}} & \multicolumn{2}{|c|}{ Cultivar } & \multirow[t]{2}{*}{ Mean } & \multicolumn{2}{|c|}{ Cultivar } & \multirow[t]{2}{*}{ Mean } & \multicolumn{2}{|c|}{ Cultivar } & \multirow[t]{2}{*}{ Mean } \\
\hline & & $\begin{array}{c}\text { Giza } \\
(\mathbf{1 0 2})\end{array}$ & $\begin{array}{c}\text { Solala } \\
(120)\end{array}$ & & $\begin{array}{l}\text { Giza } \\
(102)\end{array}$ & $\begin{array}{c}\text { Solala } \\
(\mathbf{1 2 0})\end{array}$ & & $\begin{array}{c}\text { Giza } \\
(102)\end{array}$ & $\begin{array}{c}\text { Solala } \\
(120)\end{array}$ & & $\begin{array}{c}\text { Giza } \\
(102)\end{array}$ & $\begin{array}{c}\text { Solala } \\
(120)\end{array}$ & \\
\hline \multirow{4}{*}{$60 \%$} & Control & 49.3 & 55.6 & 52.4 & 48.3 & 52.1 & 50.2 & 612 & 1026 & 819 & 541 & 923 & 732 \\
\hline & Potassium & 50.9 & 57.3 & 54.1 & 49.7 & 55.4 & 52.6 & 808 & 1222 & 1015 & 730 & 1112 & 921 \\
\hline & Kaolin & 50.2 & 56.5 & 53.3 & 49.4 & 55.1 & 52.3 & 744 & 1167 & 956 & 679 & 1061 & 870 \\
\hline & Ascorbic & 49.4 & 55.7 & 52.6 & 49.0 & 54.6 & 51.8 & 710 & 1124 & 917 & 638 & 1020 & 829 \\
\hline Mean & & 49.9 & 56.3 & 53.1 & 49.1 & 54.3 & 51.7 & 719 & 1135 & 927 & 647 & 1029 & 838 \\
\hline \multirow{4}{*}{$80 \%$} & Control & 55.1 & 61.2 & 58.1 & 53.7 & 59.4 & 56.6 & 932 & 1313 & 1123 & 828 & 1210 & 1019 \\
\hline & Potassium & 56.5 & 62.8 & 59.7 & 54.9 & 60.6 & 57.8 & 1095 & 1509 & 1302 & 1017 & 1399 & 1208 \\
\hline & Kaolin & 55.8 & 62.1 & 58.9 & 54.6 & 60.3 & 57.5 & 1040 & 1454 & 1247 & 966 & 1348 & 1157 \\
\hline & Ascorbic & 55.0 & 61.3 & 58.1 & 54.2 & 59.9 & 57.1 & 801 & 1411 & 1106 & 925 & 1307 & 1116 \\
\hline Mean & & 55.6 & 61.9 & 58.7 & \begin{tabular}{|l|}
54.4 \\
\end{tabular} & 60.1 & 57.2 & 967 & 1422 & 1194 & 934 & 1316 & 1125 \\
\hline \multirow{4}{*}{$100 \%$} & Control & 55.8 & 61.6 & 58.7 & 54.8 & 60.5 & 57.7 & 1028 & 1377 & 121 & 828 & 1263 & 1046 \\
\hline & Potassium & 57.0 & 62.8 & 59.9 & 56.2 & 61.9 & 59.1 & 1159 & 1573 & 1366 & 1017 & 1452 & 1235 \\
\hline & Kaolin & 56.2 & 62.5 & 59.4 & 55.8 & 61.5 & 58.7 & 1104 & 1518 & 1311 & 966 & 1401 & 1184 \\
\hline & Ascorbic & 55.4 & 61.8 & 58.6 & 55.5 & 61.2 & 58.4 & 1061 & 1475 & 1268 & 925 & 1360 & 1143 \\
\hline Mean & & 56.1 & 62.2 & 59.1 & 55.6 & 61.3 & 58.4 & 1088 & 1486 & 1016 & 934 & 1369 & 1152 \\
\hline \multicolumn{14}{|l|}{ LSD } \\
\hline V & & & & 0.05 & & & 0.49 & & & 47.00 & & & 45.00 \\
\hline Ir & & & & 0.03 & & & 0.51 & & & 48.00 & & & 46.00 \\
\hline$V^{*}$ ir & & & & 0.04 & & & 0.32 & & & 40.00 & & & 37.00 \\
\hline $\operatorname{Tr}$ & & & & 0.02 & & & 0.55 & & & 41.00 & & & 38.00 \\
\hline Tr*ir & & & & 0.03 & & & 0.09 & & & n.s & & & n.s \\
\hline $\mathrm{T}^{*} \mathrm{~V}$ & & & & 0.04 & & & 0.08 & & & n.s & & & n.s \\
\hline Tr*ir*V & & & & 0.06 & & & 0.09 & & & n.s & & & n.s \\
\hline
\end{tabular}




\section{Evaluation of Some Transpiration Regulators in Sunflower for Saving Irrigation Water in Relation to Productivity}

\subsection{Seed yield/fed. $(\mathrm{kg})$}

Data presented in (Table 4) indicated that seed yield/fed. was significantly affected by irrigation levels in the two seasons. Irrigation at $100 \%$ of $\mathrm{Et}_{0}$ gave the highest seed yield/ fed., whereas the lowest seed yield value was achieved by the limited watered treatment ( $60 \%$ of $\mathrm{Et}_{0}$ level). Interpretation of such finding was reported by Hall et al (1990), Human et al (1990) they found that the water stress conditions decrease photosynthesis and respiration, and as a result overall production of the crop is decreased. In comparison to the control, foliar spraying of transpiration regulators especially with potassium under $60 \%$ of $\mathrm{Et}_{0}$ irrigation level caused significant increase in seed yield during the two growing seasons. These results are in harmony with those observed by Gaballah et al (2014) who study the influence of transpiration regulators like potassium, ascorbic an alkaloid and organic compost on sunflower yield and yield quality under sandy soil condition and found that, KCL was the best antitranspirant compared the other used and its efficiency increase by using organic compost under the sandy soil conditions. Similar results were obtained by (Peter 2008).

Significant difference in seed yield ( $\mathrm{kg} /$ fed.) was found between the two used cultivars in both seasons. Solala 120 gave higher seed yield value and exceeded those obtained by Giza 102 cultivar by 36.6 and $46.6 \%$ in 2015 and 2016 seasons, respectively. As for interaction among the studied factors, results showed that there were no significant effects on seed yield/fed. due to these interactions.

\subsection{Seed oil \%}

Data in (Table 5) show that irrigation at $100 \%$ of $\mathrm{Et}_{0}$ produced the highest seed oil content $\%$ in both seasons. Otherwise, seed oil percentage under sever water limitation $(60 \%$ of $\mathrm{Et}_{0}$ ) was considerably lower than under other treatments $\left(80\right.$ and $100 \%$ of $\left.\mathrm{Et}_{0}\right)$. This results are in harmony with that of Poudineh et al (2015) who showed that the highest value of seed oil were obtained with optimum irrigation and there was no significant difference between the mild stress $80 \%$ of $\mathrm{Et}_{0}$ and sever stress $60 \%$ of $\mathrm{Et}_{0}$. Similar trend was obtained by Sullu and Dagdelen (2015). Comparative differences between the three types of transpiration regulators (potassium, kaolin and ascorbic) and the control on seed oil content $\%$ were presented in (Table 5) where Potassium followed by Kaolin were the effective in increasing significantly seed oil\% value. In this respect Gaballa et al (2014) showed that spraying sunflowerplants with KCL as transpiration regulators at rates of 5 and $10 \mathrm{~g} / \mathrm{L}$ significantly increased seeds yield and oil yield by 52.7 and $61.7 \%$ and 91.6 and $111.2 \%$ than that of untreated, respectively.

Results in (Table 5) illustrated that sunflower seed oil\% value was governed significantly by cultivars. Seed oil \% value of Solala 120 cultivar was higher than that of Giza 102 cultivar. Ptency of Solala 120 in this respect was significant in both the two experimental seasons.

\subsection{Oil yield (kg/fed)}

Oil yield $(\mathrm{kg} / \mathrm{fed})$ was significantly decreased as water irrigation requirement decreased from 100 to $60 \%$ of irrigation at both seasons as in (Table 5). These results are in parallel line with those obtained by (Attia et al 1998, Abbas and Anton 1999). There were significant differences between the two tested cultivars. Solala 120 gave high oil yield (kg/fed.) value as compared to Giza 102. Such trend was fact in the both seasons of 2015 and 2016. This is may due to the superiority of Solala 120 in seed yield $\mathrm{kg} / \mathrm{fed}$. as well as in oil\%. Interaction between irrigation levels and antitranspirant agents had significant effects on oil yield (kg/fed.). Combination between $100 \%$ of $\mathrm{Et}_{0}$ and Potassium antitranspirant agent significantly increased oil yield. This was true in two seasons, compared to the control (Table 5). Bieloria and Hopmans (1975) found that drought stress limited the carbohydrate supply for grains of sunflower plants via 
stomata closure, reduction in leaf area and photosynthesis as well as shortening of the grain filling period.

\subsection{Total chlorophyll content}

The results given in (Tables 6) showed the effect of irrigation levels and transpiration regulators (potassium, kaolin and ascorbic) on chlorophyll content of leaves of sunflower cultivars during the two seasons of 2015 and 2016. It is clear from the tabulated data that, chlorophyll content (total chl.) at flowering stage were higher under watered with $100 \%$ of $\mathrm{Et}_{0}$. on the other hand, $60 \%$ of $\mathrm{Et}_{0}$ recorded the lowest value of such trait in the two growing seasons. In most stressed plants, the decrease in chlorophyll content may be attributed to thylakoid membrane disorganization, with more degradation than chlorophyll synthesis by proteolytic enzyme formation. This is responsible for the degradation of chlorophyll and damage to the photosynthetic apparatus (Rong-hua et al 2006). On the other hand, Ripley et al (2007) proved that water deficit may decrease photosynthetic assimilation by both stomatal and metabolic limitations.

Results of the present investigation cleared that the application of transpiration regulators had exerted a profound improving impact on total chlorophyll values compared with untreated (control) plants. Potassium transpiration regulator treatment exhibited the highest value in this respect. This finding might be attributed to the fruitful influence of transpiration regulators in favoring water status of stressed plant tissues and maintain chlorophyll molecules synthesis. In this concern, Adolfo (2007) propagated that the beneficial effect of applied transpiration regulators might be due to the simulative effects on photosynthetic capacity by overcoming stomata limitations, protecting photosynthetic pigments from water shortage induced degradation or enhancing biosynthesis of photosynthetic pigments.

Total chlorophyll content of sunflower leaves was judged by cultivar used (Giza 120 and Solala 102). Superiority in this respect also still remains with Solala 120 cultivar. Impact of interaction between the two factors on chlorophyll content of sunflower leaves was not great enough to reach the 5\% significance level (Table 6).

\subsection{Water use efficiency (WUE)}

Water use efficiency of the two sunflower cultivars expressed as $\mathrm{kg}$ seeds produced per $\mathrm{m}^{3}$ of water consumed in complete evapotranspiration as affected by irrigation levels and transpiration regulators treatments for 2015 and 2016 seasons are presented in (Table 6). Results showed that WUE values for irrigation levels were significantly differed in both seasons. Irrigation at $60 \%$ of $\mathrm{Et}_{0}$ resulted high WUE value as compared with irrigation at 80 , $100 \%$ of $\mathrm{Et}_{0}$. Vites (1965) found that water use efficiency is not clearly depend on the water available and evapotranspiration limit, even the crop yield and the opportunity to increase it do depend on the adequancy of water supply. Similar results on cotton were obtained by (Dawood 2006). Concerning cultivars, data revealed that the WUE for Solala 102 was significantly higher than for Giza 102 in the two seasons. Reading transpiration regulators results showed significant effect of the tested treatments on WUE values. All transpiration regulators surpassed the control in both seasons. All interactions among factors under study due to WUE were not significant in 2015 and 2016 seasons. 


\section{Evaluation of Some Transpiration Regulators in Sunflower for Saving Irrigation Water in Relation to Productivity}

Table 5. Effect of irrigation regimes and transpiration regulators on seed oil content \% and oil yield $(\mathrm{kg} / \mathrm{fed})$ of sunflower cultivars in 2015 and 2016 seasons

\begin{tabular}{|c|c|c|c|c|c|c|c|c|c|c|c|c|c|}
\hline \multirow{4}{*}{ Irrigation } & \multirow{4}{*}{ Treatments } & \multicolumn{6}{|c|}{ Seed oil content \% } & \multicolumn{6}{|c|}{ Oil yield kg /fed } \\
\hline & & \multicolumn{3}{|c|}{$1^{\text {st }}$ season $(2015)$} & \multicolumn{3}{|c|}{$2^{\text {nd }} \operatorname{season}(2016)$} & \multicolumn{3}{|c|}{$1^{\text {st }}$ season $(2015)$} & \multicolumn{3}{|c|}{$2^{\text {nd }} \operatorname{season}(2016)$} \\
\hline & & \multicolumn{2}{|c|}{ Cultivar } & \multirow[t]{2}{*}{ Mean } & \multicolumn{2}{|c|}{ Cultivar } & \multirow[t]{2}{*}{ Mean } & \multicolumn{2}{|c|}{ Cultivar } & \multirow[t]{2}{*}{ Mean } & \multicolumn{2}{|c|}{ Cultivar } & \multirow[t]{2}{*}{ Mean } \\
\hline & & $\begin{array}{l}\text { Giza } \\
(102)\end{array}$ & $\begin{array}{c}\text { Solala } \\
(\mathbf{1 2 0})\end{array}$ & & $\begin{array}{l}\text { Giza } \\
(102)\end{array}$ & $\begin{array}{c}\text { Solala } \\
(120)\end{array}$ & & $\begin{array}{c}\text { Giza } \\
(102)\end{array}$ & $\begin{array}{c}\text { Solala } \\
(120)\end{array}$ & & $\begin{array}{c}\text { Giza } \\
(102)\end{array}$ & $\begin{array}{c}\text { Solala } \\
(120)\end{array}$ & \\
\hline \multirow{4}{*}{$60 \%$} & Control & 40.2 & 43.4 & 41.8 & 39.7 & 43.2 & 41.45 & 300.2 & 400.2 & 350.2 & 299.7 & 379.3 & 339.5 \\
\hline & Potassium & 41.3 & 43.5 & 42.4 & 41.1 & 43.3 & 42.2 & 382.2 & 482.2 & 432.2 & 375.7 & 455.4 & 415.6 \\
\hline & Kaolin & 40.4 & 42.6 & 41.5 & 40.0 & 42.4 & 41.2 & 357.6 & 457.6 & 407.6 & 353.2 & 412.9 & 383.05 \\
\hline & Ascorbic & 40.3 & 42.1 & 41.2 & 39.7 & 42.2 & 41.0 & 348.9 & 448.9 & 398.9 & 333.2 & 400.1 & 366.7 \\
\hline Mean & & 40.6 & 42.9 & 41.7 & 40.1 & 42.8 & 41.5 & 347.2 & 447.2 & 397.2 & 340.5 & 411.925 & 376.2 \\
\hline \multirow{4}{*}{$80 \%$} & Control & 41.5 & 44.7 & 43.1 & 40.2 & 43.2 & 41.7 & 394.9 & 600.3 & 497.6 & 346.6 & 535.7 & 441.2 \\
\hline & Potassium & 42.4 & 44.5 & 43.45 & 42.2 & 44.1 & 43.2 & 476.9 & 682.7 & 579.8 & 422.7 & 617.7 & 520.2 \\
\hline & Kaolin & 41.3 & 43.7 & 42.5 & 41.4 & 43.3 & 42.4 & 451.9 & 657.7 & 554.8 & 400.1 & 539.2 & 469.7 \\
\hline & Ascorbic & 41.2 & 42.3 & 41.75 & 41.2 & 42.2 & 41.7 & 443.6 & 649.0 & 546.3 & 380.2 & 584.4 & 482.3 \\
\hline Mean & & 41.6 & 43.8 & 42.7 & 41.3 & 43.2 & 42.2 & 441.8 & 647.4 & 544.6 & 387.4 & 569.3 & 478.3 \\
\hline \multirow{4}{*}{$100 \%$} & Control & 45.0 & 46.1 & 45.6 & 43.7 & 45.7 & 44.7 & 436.3 & 641.9 & 539.1 & 387.9 & 577.0 & 482.5 \\
\hline & Potassium & 46.6 & 48.0 & 47.3 & 46.1 & 46.8 & 46.5 & 518.4 & 723.9 & 621.2 & 464.0 & 653.1 & 558.6 \\
\hline & Kaolin & 43.7 & 44.5 & 44.1 & 43.3 & 44.4 & 43.9 & 498.0 & 699.3 & 598.7 & 441.5 & 630.6 & 536.1 \\
\hline & Ascorbic & 43.2 & 44.3 & 43.75 & 43.2 & 44.2 & 43.7 & 485.4 & 690.6 & 690.6 & 421.5 & 610.6 & 516.1 \\
\hline Mean & & 44.6 & 45.7 & 45.2 & 44.1 & 45.3 & 44.7 & 484.5 & 688.9 & 586.7 & 428.7 & 617.8 & 523.3 \\
\hline \multicolumn{14}{|l|}{ LSD } \\
\hline \multicolumn{14}{|l|}{ V } \\
\hline Ir & & & & 0.02 & & & 0.05 & & & 24.28 & & & 14.33 \\
\hline $\mathrm{V}^{*}$ ir & & & & 0.06 & & & 0.08 & & & 18.98 & & & 10.77 \\
\hline $\operatorname{Tr}$ & & & & 0.08 & & & 0.12 & & & 26.84 & & & 24.81 \\
\hline Tr*ir & & & & 0.06 & & & 0.04 & & & 19.80 & & & 13.56 \\
\hline $\mathrm{T} * \mathrm{~V}$ & & & & 0.08 & & & 0.05 & & & n.s & & & n.s \\
\hline \multirow[t]{2}{*}{ Tr*ir*V } & & & & 0.10 & & & 0.07 & & & n.s & & & n.s \\
\hline & & & & 0.14 & & & 0.93 & & & n.s & & & n.s \\
\hline
\end{tabular}

V: Cultivar IR: irrigation levels

Tr: treatments 
Table 6. Effect of irrigation and transpiration regulators on total chlorophyll and water use efficiency of two sunflower cultivars in 2015 and 2016 seasons

\begin{tabular}{|c|c|c|c|c|c|c|c|c|c|c|c|c|c|}
\hline \multirow{4}{*}{ Irrigation } & \multirow{4}{*}{ Treatments } & \multicolumn{6}{|c|}{ Total chlorophyll (mg/g) } & \multicolumn{6}{|c|}{ Water use efficiency ( WUE,kg/m3/fed) } \\
\hline & & \multicolumn{3}{|c|}{$1^{\text {st }}$ season (2015) } & \multicolumn{3}{|c|}{$2^{\text {nd }} \operatorname{season}(2016)$} & \multicolumn{3}{|c|}{$1^{\text {st }}$ season $(2015)$} & \multicolumn{3}{|c|}{$2^{\text {nd }} \operatorname{season}(2016)$} \\
\hline & & \multicolumn{2}{|c|}{ Cultivar } & \multirow[t]{2}{*}{ Mean } & \multicolumn{2}{|c|}{ Cultivar } & \multirow[t]{2}{*}{ Mean } & \multicolumn{2}{|c|}{ Cultivar } & \multirow[t]{2}{*}{ Mean } & \multicolumn{2}{|c|}{ Cultivar } & \multirow[t]{2}{*}{ Mean } \\
\hline & & $\begin{array}{c}\text { Giza } \\
(102)\end{array}$ & $\begin{array}{c}\text { Solala } \\
(\mathbf{1 2 0})\end{array}$ & & $\begin{array}{c}\text { Giza } \\
(\mathbf{1 0 2})\end{array}$ & $\begin{array}{r}\text { Solala } \\
(120)\end{array}$ & & $\begin{array}{c}\text { Giza } \\
(\mathbf{1 0 2})\end{array}$ & $\begin{array}{c}\text { Solala } \\
(120)\end{array}$ & & $\begin{array}{l}\text { Giza } \\
(102)\end{array}$ & $\begin{array}{c}\text { Solala } \\
(120)\end{array}$ & \\
\hline \multirow{4}{*}{$60 \%$} & Control & 3.35 & 3.50 & 3.42 & 3.23 & 3.38 & 3.31 & 876 & 899 & 888 & 885 & 905 & 895 \\
\hline & Potassium & 3.76 & 3.91 & 3.84 & 3.62 & 3.77 & 3.69 & 833 & 857 & 845 & 841 & 863 & 852 \\
\hline & Kaolin & 3.57 & 3.75 & 3.66 & 3.44 & 3.59 & 3.51 & 823 & 838 & 831 & 830 & 844 & 837 \\
\hline & Ascorbic & 3.45 & 3.62 & 3.53 & 3.34 & 3.49 & 3.42 & 811 & 817 & 814 & 818 & 823 & 821 \\
\hline Mean & & 3.53 & 3.69 & 3.61 & 3.41 & 3.56 & 3.48 & 836 & 853 & 844 & 844 & 859 & 851 \\
\hline \multirow{4}{*}{$80 \%$} & Control & 3.78 & 3.91 & 3.845 & 3.53 & 3.66 & 3.595 & 1301 & 1271 & 1286 & 1308 & 1277 & 1293 \\
\hline & Potassium & 4.18 & 4.31 & 4.24 & 3.92 & 0.46 & 2.19 & 1224 & 1195 & 1210 & 1230 & 1211 & 1221 \\
\hline & Kaolin & 3.98 & 4.11 & 4.05 & 3.74 & 3.87 & 3.80 & 1207 & 1172 & 1190 & 1213 & 1178 & 1196 \\
\hline & Ascorbic & 3.91 & 4.00 & 3.96 & 3.64 & 3.77 & 3.71 & 1135 & 1105 & 1120 & 1141 & 1120 & 1131 \\
\hline Mean & & 3.96 & 4.08 & 4.02 & 3.71 & 2.94 & 3.32 & 1217 & 1186 & 1201 & 1223 & 1197 & 1210 \\
\hline \multirow{4}{*}{$100 \%$} & Control & 3.73 & 4.37 & 4.05 & 4.02 & 4.13 & 4.08 & 1669 & 1586 & 1628 & 1686 & 1598 & 1642 \\
\hline & Potassium & 4.67 & 4.78 & 4.73 & 4.32 & 4.52 & 4.42 & 1578 & 1461 & 1520 & 1584 & 1473 & 1529 \\
\hline & Kaolin & 4.48 & 4.59 & 4.53 & 4.25 & 4.34 & 4.296 & 1517 & 1451 & 1484 & 1532 & 1463 & 1498 \\
\hline & Ascorbic & 4.37 & 4.48 & 4.43 & 4.14 & 4.25 & 4.19 & 1492 & 1357 & 1425 & 1507 & 1378 & 1443 \\
\hline Mean & & 4.31 & 4.55 & 4.43 & 4.18 & 4.31 & 4.25 & 1564 & 1464 & 1514 & 1577 & 1478 & 1528 \\
\hline \multicolumn{14}{|l|}{ LSD } \\
\hline V & & & & 0.160 & & & 0.021 & & & 0.002 & & & 0.002 \\
\hline $\mathrm{Ir}$ & & & & 0.130 & & & 0.017 & & & 0.003 & & & 0.001 \\
\hline$V^{*}$ ir & & & & 0.027 & & & 0.025 & & & 0.005 & & & 0.001 \\
\hline $\operatorname{Tr}$ & & & & 0.130 & & & 0.018 & & & 0.002 & & & 0.002 \\
\hline Tr*ir & & & & 0.190 & & & 0.025 & & & 0.002 & & & 0.003 \\
\hline $\mathrm{T}^{*} \mathrm{~V}$ & & & & 0.230 & & & 0.031 & & & 0.003 & & & 0.003 \\
\hline $\mathrm{Tr}^{*} \mathrm{ir}^{*} \mathrm{~V}$ & & & & 0.320 & & & 0.043 & & & 0.004 & & & 0.005 \\
\hline
\end{tabular}

\section{Conclusion}

In the light of the present study, water stress had a negative impact on growth parameters, yield and yield components and oil percentage and its yield in $\mathrm{kg} / \mathrm{fed}$. of sunflower plants at $60 \%$ of $\mathrm{Et}_{0}$ stress in comparison to the optimum irrigation $\left(100 \%\right.$ of $\left.\mathrm{Et}_{0}\right)$ level. Whereas mild-watered $\left(80 \%\right.$ of $\left.\mathrm{Et}_{0}\right)$ treatment was inbetween. The use of Potassium may be helpful tool to mitigate the negative effects of water stress and to improve the water use efficiency in sunflower plants under water deficit conditions. 


\section{Evaluation of Some Transpiration Regulators in Sunflower for Saving Irrigation Water in Relation to Productivity}

\section{References}

AOAC (1980) Asociation of Official Agricultural Chemists. Official Methods of Analysis. $13^{\text {th }}$ Ed., Washington, D.C., USA.

Abbas FA, Anton NA (1999) Effect of irrigation intervals on sunflower production. $3^{\text {rd }}$ Conf. On Farm Irrigation and Agroclimatology 1, 633-646.

Abdel-fatah GH (2013) Response of water stressed rose of China (Hibiscus rosa-sinensis L.) plant to treatment with calcium carbonate and vapor gardantitranspirants. J Appl Sci Res 9, 3566-3572.

Abdel-Gawad AA, Ashoub MA, Saleh SA, ElGazar MM(1987) Yield response of some sunflower cultivars to irrigation intervals. Ann Agric Sci, Fa. of Agric, Ain Shams Univ 32, 1229-1242.

Adolfo. Rosati, (2007) Physiological Effects of Kaolin Particle Film Technology: Review. Functional Plant Science and Biotechnology Global Science Books, pp 100-105.

Ahmed S, Ahmed R, Ashraf M Y, Ashraf M, Waraich EA (2009) Sunflower (Helianthus annuus L.) response to drought stress at germination and seedling growth stages. Pak J Bot 41, 647-654.

Anjum SA, Xi X, Wang Saleem MF, Man C, Lei W (2011) Morphological, physiological and biochemical responses of plants to drought stress. African J Agric Res 6, 2026-2032.

Albert A, Schneiter ED (1997). Sunflower Tecnology and Production. Madison, Wisconsin: The American Society of Agronomy, USA, pp 595-670.

Attia SA, El-Refaey RA, El-Hity MA (1998) Effect of irrigation regimes on two varietiesof sunflower (Helianthus annuus L.) at North Delta region. Agron J Kafr El-Sheikh 11, 162171.
Attia SAM (1985) Effect of some cultural treatments on sunflower (Helianthus annuus L.). Ph.D. Thesis Fac of Agric Alazhar Univ, Egypt.

Badr NM, Mohamed MH (2004) Effect of foliar spraying with the nutrient compound "streen on the growth and yield of sunflower plants subjected to water stress during various stages of growth. Bull the Nati Res Cen Cairo 29, 427-439.

Baldini M, Vannozzi GP (1999) Yield relationships under drought in sunflower genotypes obtained from a wild population and cultivated sunflower in rain-outshetter in large post and field experiments. Helia 22, 81-96.

Barrs HD, Weatherly PEC (1962) Examination of relative turgidity technique for estimating water deficits in leaves. Aust J Bio Sci 15, 413-428.

Bieloria H, Hopmans PAM (1975) Recovery of leaf water potential, transpiration and photosynthesis of cotton during irrigation cycles. Agronomy J 67, 629-632.

Conde A D, Pimentel A, Neves, Dinis L, Bernardo S, Correia CM, Geros H, Moutinhopereira J (2016) Kaolin foliar application has a stimulatory effect on phenylpropanoid and flavonoid pathways in grape berries. Front. Plant Sci 7, 1150.

Dawood AMM (2006) Response of some Egyptian cotton varieties to biofertilization and irrigation intervals in the newly reclaimed land of west Nubaria. Ph.D. Thesis, Fac of Agri. (Saba Basha). Alex Univ, Alex, Egypt.

Demian KR (1999) Effect of some agronomic practice on seed and oil yield of sunflower grown in calcareous soil. Ph.D. Thesis Fac of Agric, Kafr El-sheikh, Tanta Univ, Tanta, Egypt.

El-Ahmer BA, El-Mohandes SI, Madkour, MA (1989) Variation and interrelationships of some characters in sunflower. Assiut J of Agric Sci 20, 327-327. 
El-Hity MA, Zahran M, El-Aishy SM, El-Zayat M (1994) Effect of plant population density and nitrogen rate on two sunflower cultivars. II-yield and its components. J Agric Res 20, 480- 489.

El-Mantawy R F, Khedr R A, El-Hag DA (2017) Response of sunflower (Helianthus annuus $\mathrm{L}$.) to zinc sulfate spraying under deficit irrigation at different growth stages. $J$ Plant Archives 17, 24-33.

El-Sharkawy HMM, Salama FM (1973) Response of olive and almond orchards to partial irrigation under dry farming practices in semi arid regions. II- plant soil water relations in olive during the growing season. Plant and Soil 41, 13-32.

El-Temsah MH (2008) Effect of irrigation, mineral and biofertization on yield and yield components of sunflower. Ph.D. Thesis, Fac. of Agric. Saba Basha, Alex. Univ.

Gaballah MS, Shaaban SM, Abdallah EF (2014) The use of antitranspirants and organic compost in sunflower grown under water stress sandy soil. International Journal of Acadimic Research, 6 p.

Hall AJ, Conner DJ, Whitifield DM (1990) Root respiration during grain filling in sunflower: The effect of water stress. Plant and Soil 121, 57-66.

Hoque TS, Hossain MA, Mostofa MG, Burritt DJ, Fujita M, Tran LSP (2016) Methylglyoxal: An emerging signaling molecule in plant abiotic stress responses and tolerance. Plant Sci 7 , 1341.

Human, JJD, Bezuidenhout, HD, Bruyn, LP (1990) The influence of plant water stress on net photosyntheasis and yield of sunflower. Crop Sci, 164, 231-241.

Ismail AE, Hasabo SA (2000) Evaluation of some new Egyptian commercial biofertilizers, plant nutrients and biocid against Meloidogy incognita root knot nematode infecting sunflower. PakJ Nemat 18, 39-49.
Israelsen OW, Hansen VE (1962) Irrigation Principles and Practices. The $3^{\text {rd }}$ Edit, John wily and Sons Inc., New York, pp 240-253.

Jensen ME (1983) Design and Operation of Farm Irrigation System. Amer. Soc. Agri. Eng., Michigam, USA.

Johnson RE (1967) Comparison of methods for estimating cotton leaf area. Agron J 59, 493-494.

Karam F, Randa M, Rabih K, Joelle B, Claude C, Yossef R (2007) Evapotranspiration, seed yield and water use efficiency of drip irrigated sunflower under full and deficit irrigations. Agricultural Water Magement 90, 213-223.

Khan AM, Iqbal M, Ahmed I, Iqbal N, Hussain M (2000) Effect of different water stress levels on yield and oil content of sunflower (Helianthus annuus L.) cultivars. Pak J Biol Sci 3, 1632-1633.

Knowles PF (1988) Recent advances in oil crops breeding In: Applewhite, TH (ed.) Proceedings of the World Conference on Biotechnology for the Fats and Oil Industry, Urbana, Illinois: American Oil Chemists Society.pp 3538.

Leon AJ, Andrade FH, Lee M (2003) Genetic analysis of seed oil concentrations across generations and environments in sunflower (Helianthus annuus L.). Crop Science 43, 135-140.

Liux Baird WV (2003) Differential expression of genes regulated in response to drought or salinity stress in sunflower. Crop Science 43, 678-687.

Lopez-Pereira M, Trapani N, Sadras V (2000) Genetic improvement of sunflower in Argentina between 1930 and 1995. III. Dry matter partitioning and achene composition. Field Crops Research 67, 215-221.

Miller JF (1995) Inheritance of salt tolerance in sunflower (Helianthus annuus L.) Helia 18, $9-16$. 


\section{Evaluation of Some Transpiration Regulators in Sunflower for Saving Irrigation Water in Relation to Productivity}

Monoroy SS, Vallejo AU, Gody AR, Diaz HR (2015) Effect of Kaolin application on growth, water use efficiency, and leaf epidermis characteristics of (Physalis peruviana $\mathrm{L}$ ). seedlings under two irrigation regimes. J Agric Sci Tech $17,1585-2596$.

Moran R (1982) Formula determination of chlorophylls pigements extracted with N-N dimethyl- frmamide. Plant Physiol 69, 13761381.

Nasraui B (1993) Role of antitranspirant films in protecting plants against fungal disease. Annals de linstitute National de la Recherch Agronomique de Tunisie 66, 125-135.

Ouda SA, El-Mesiry T, Gaballah MS (2007) Effect of using stabilizing agents on increasing yield and water use efficiency in barley grown under water stress. Australian Journal of Basic and Applied Science 1, 571-577.

Peter, KV (2008) Basic of Horticulture.New India Publ. Ageny. pp 50-60.

Poudineh Z, Moghadam ZG, Mirshekari S (2015) Effects of humic acid and folic acid on sunflower under drought stress. Bio Forum- An Inter J 7, 451-454.

Prakash M, Ramachandran K (2000) Effects of Chemical Ameliorants in Brinjal (Solanum melongena L.) under Moisture Stress Conditions. J Agro Crop Sci 185, 237-239.

Prunty L (1983) Soil water and population influence on hybrid sunflower yield and uniformity of stand. Agron J 75, 745-749.

Rady GKM, Dangi KS, Kumar SS, Reddy AV (2003) Effect of moisture stress on seed yield and quality in sunflower (Helianthus annuus L.). J Oil Res 20, 282-283.

Rauf S, Sadaqate HA (2007) Sunflower (Helianthus annuus L.) germoplasm evaluation for drought tolerance. Comm Bio and Crop Sci 2, 8-16.

Ripley BS, Gilbert ME, Ibrahim DG, Osborne CP (2007) Drought constraints on $\mathrm{C}_{4}$ photosynthesis: stomatal and metabolic limita- tions in $\mathrm{C}_{3}$ and $\mathrm{C}_{4}$ subspecies of Alloteropsissemialata. $J$ of Experimental Botany 58,1351 1363.

Rong-Hua L, Pei-guo G, Baum M, Grando S, Ceccarelli S (2006) Evaluation of chlorophyll content and fluorescence as indicators of drought tolerance in barley. Agricultural Sciences in China 5, 751-757.

Salera E, Detti GM (1992) Adaptation and yield potential of sunflowers on the inner Tuscan hills. Field Crop Abst 4841.

Sherif- Faiza MA (2002) Effect of biofertiliztion on the yield and quality of sunflower in relation to nitrogen fertilization. Ph.D. Thesis, Fac of Agric (Saba Basha). Alex Univ, Alex, Egypt.

Steel RGD, Torrie JH (1980) Principles and Procedures of Statistics, 2nd Ed., McGrawHill Co., New York, USA.

Stockle CO, Kiniry KR (1990) Variability in crop radiation- use efficiency associated with vapour- pressure deficit. Field Crop Res 25, 171-181.

Sullu A, Dagdelen N (2015) The evaluation of drip irrigation on second crop sunflower yield and quality in Soke region. Journal of Adnan Menderess University Agricultural Faculty 12, 45-54.

Umar S, Moinuddin M (2002). The effect of sources and rates of potassium application on potato yield and economic returns. Better Crop Int 15:13-15.

Unyayar S, Keles Y, UnalE (2004) Proline and ABA levels in two sunflower genotypes subjected to water stress. Bulg J Plant Physiol 30, 34-47.

Vites FGJr (1965) Increasing water use efficiency by soil management. Agron Madision Wisc, 259-274.

Wahba SA, Adel Rahman SI, Tayel MY, Matyn MA (1990) Soil moisture, salinity, water use efficiency and sunflower growth as influenced by irrigation, bitumen mulch and plant density. Soil Tech 3, 33-44. 
Weatherly, PE (1950) Studies on the water relations of the cotton plants. I. The field measurement of water deficits in leaves. New Phytol 49, 81-97.
Yegappan TM, Paton DM, Gates CT, Muller WJ (1982) Water stress in sunflower (Helianthus annus L.) effect on leaf. Ann Bot 49, 6368. 